\title{
Sustainable development through Internet of Things
}

\section{R. Maheswar ${ }^{1}$ - G. R. Kanagachidambaresan ${ }^{2}$}

Published online: 3 February 2020

(C) Springer Science+Business Media, LLC, part of Springer Nature 2020

The needs of sustainable development of society are satisfied through Artificial Intelligence and miniaturized computers. The requirement of people are increasing exponentially and satisfying the same with generalized framework is a challenging task. The Internet of Things (IoT) provides more sophistication with limited resource. The sustainable development areas like, smart healthcare, smart agriculture, smart transportation is met through deep learning and next generation communication networks. The sustainable development faces challenges on sensor design, communication delay and security challenges in hardware and software side. All these issues are mainly satisfied through design and implementation perspective view. This special issue focuses on state of art including protocol design and low cost sensor design for sustainable development of society using Internet of Things (IoT).

This special issue features 8 selected high quality research articles. The first paper "Dynamic Clustering Approach Based on Wireless Sensor Networks Genetic Algorithm for IoT Applications" explores dynamic clustering based methodology and frame relay nodes are improved to elect the most preferred sensor node. Simulation results elucidates the proposed algorithm provides better results. The second article entitled "A Novel Free Space Communication System Using Nonlinear InGaAsPMicrosystem Resonators for Enabling PowerControl Toward Smart Cities" provides 10 times bandwidth improvement over the radius of $600 \mathrm{~m}$. The overall proposed system will able to detect FSO signal and extract original data. The third article "Genetic Algorithm based

R. Maheswar

maheshh3@rediffmail.com

G. R. Kanagachidambaresan

kanagachidambaresan@gmail.com

1 School of EEE, VIT Bhopal University, Bhopal, India

2 Department of CSE, Vel Tech Rangarajan Dr. Sagunthala R\&D Institute of Science and Technology, Avadi, Chennai, India
Adaptive Offloading for Improving IoT Device Communication Efficiency" introduces genetic algorithm based adaptive offloading (GA-OA) for effective traffic handling in IoT-infrastructure-cloud environment. The fourth article "A Trust Computed Framework for IoT Devices and Fog Computing Environment" demonstrates the transmission processing concerns of fog nodes and IoT device layer attack during the handoff (mobility) of IoT devices in the fog environment. A secure routed and handoff mechanism is proposed in order to avoid the attack by exploring the trust value and rating of each fog IoT and fog nodes/devices based on their communication behavior. The fifth article "DABPR: A Large-scale Internet of Things- based Data Aggregation Back Pressure Routing for Disaster Management" propose a data aggregation back pressure routing (DABPR) scheme, which aims to simultaneously aggregate overlapping routes for efficient data transmission and prolong the lifetime of the network. The sixth article "I-CARES: Advancing Health Diagnosis and Medication through IoT" describes a novel approach development with IoT prototype of Wireless Sensor Network and Cloud based system to provide continuous monitoring of a patient's health status. The seventh article "An Enhanced Security Mechanism through Blockchain for EPolling/ Counting Process using IoT Devices" describes an enhanced security mechanism through Blockchain in E-voting application using IoT devices where the user will create an account with proper verification done via voter id and other biometric methods on a smart device. The last article "Development of high-speed FSO transmission link for the implementation of 5G and Internet of Things" proposes the development of high-speed long-reach FSO link for the implementation of $5 \mathrm{G}$ and IoT.

Acknowledgements Our sincere and heartfelt thanks to the Editor-inChief, Dr. Imrich Chlamtac, Wireless Networks Journal, Springer and Eliška Vlčková, Managing Editor, European Alliance for Innovation (EAI) for their continuous support and guidance during the entire process. We also extend our sincere thanks and grateful to the reviewers for their efforts in reviewing the manuscripts.

Publisher's Note Springer Nature remains neutral with regard to jurisdictional claims in published maps and institutional affiliations. 


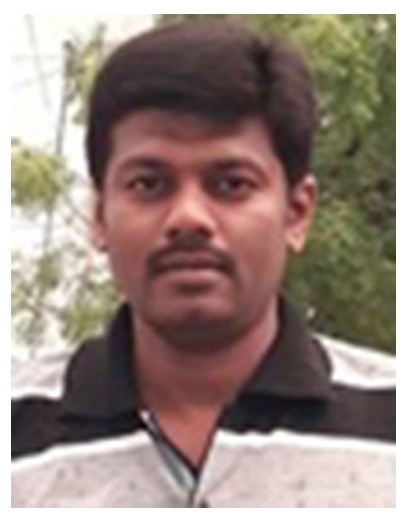

Dr. R. Maheswar has completed his B.E. (ECE) from Madras University in the year 1999, M.E. (Applied Electronics) from Bharathiyar University in the year 2002 and Ph.D. in the field of Wireless Sensor Network from Anna University in the year 2012. He has about 18 years of teaching experience at various levels and presently working as Dean-Research (Assistant) and Associate Professor in the School of EEE, VIT Bhopal University, Bhopal. He has published 60 papers at International Journals and International Conferences and published patents. His research interest includes Wireless Sensor Network, Optical Sensors, Cross Layer Design Issues, Modeling and Simulation, IoT, Queueing theory and Performance Evaluation. He has been serving as editorial board member for Wireless Networks Journal, Springer and AIHC, Springer and also edited EAI/Springer Innovations in Communications and Computing book series. He has also been serving as editorial review board member for many peer reviewed journals.

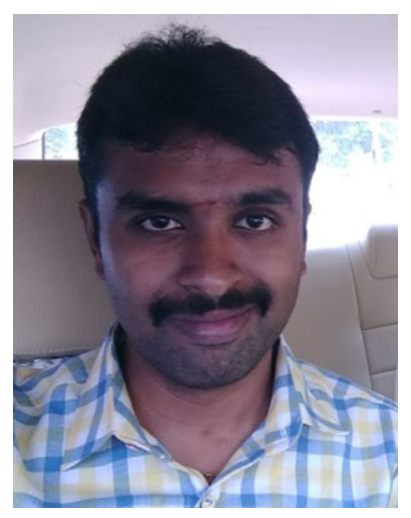

Dr. G. R. Kanagachidambaresan received his B.E. degree in Electrical and Electronics Engineering from Anna University in 2010 and M.E. Pervasive Computing Technologies in Anna University in 2012. He has completed his $\mathrm{Ph} . \mathrm{D}$. in Anna University Chennai in 2017. He is currently an Associate Professor, Department of CSE, Veltech Rangarajan Dr. Sagunthala R\&D Institute of Science and Technology. His main research interest includes Industry 4.0, Smart city projects, Body Sensor Network and Fault tolerant Wireless Sensor Network. He has published several reputed articles and undertaken several consultancy activities for leading MNC companies. He has also guest edited several special issue articles and serving as editorial review board member for peer reviewed journals. He is presently an associate editor in Wireless Networks Springer. 